\title{
pyuvsim: A comprehensive simulation package for radio interferometers in python.
}

\author{
Adam E. Lanman ${ }^{4,7}$, Bryna J. Hazelton ${ }^{1,2,7}$, Daniel C. Jacobs ${ }^{3,7}$, \\ Matthew J. Kolopanis ${ }^{3,7}$, Jonathan C. Pober ${ }^{4,7}$, James E. Aguirre ${ }^{6,7}$, \\ and Nithyanandan Thyagarajan ${ }^{5,7}$
}

1 University of Washington, eScience Institute 2 University of Washington, Physics Department 3 Arizona State University, School of Earth and Space Exploration 4 Brown University, Physics Department 5 National Radio Astronomy Observatory 6 University of Pennsylvania, Physics Department 7 Radio Astronomy Software Group

DOI: $10.21105 /$ joss.01234

\section{Software \\ - Review 七 \\ - Repository ¿ \\ - Archive ${ }^{\top}$}

Submitted: 01 February 2019 Published: 15 May 2019

\section{License}

Authors of papers retain copyright and release the work under a Creative Commons Attribution 4.0 International License (CC-BY).

\section{Summary}

Data simulations are essential to the progress of low-frequency radio telescope arrays such as HERA, the MWA, and LOFAR, and for future instruments like the SKA. Simulated datasets are used to verify analysis pipelines, to provide models for sky-based calibration, and to test the effects of design choices and environmental factors. Existing simulators (CASA, OSKAR, FHD, PRISim, et al.) make simplifying assumptions to reduce the computational demand of evaluating the measurement equation, compromising accuracy for speed. This can lead to unexpected numerical effects, which can be hard to distinguish from real effects seen in data.

pyuvsim is a simulator designed to be accurate and verifiable up front, with strict control over any approximations being made and including all effects found to be important to $21 \mathrm{~cm}$ cosmological experiments. The default behavior is to add contributions from each point source above the horizon to each baseline in a full Jones-matrix formalism (Hamaker, Bregman, \& Sault, 1996), with floating precision source positions and interpolated Efield primary beam values. Results are tested against analytic calculations whenever possible, and compared with other simulators and data for consistency, including PRISim (Thyagarajan, Kolopanis, Jacobs, Murray, \& santoshmh, 2019) and FHD (Sullivan et al., 2012). Data handling and primary beam modeling are done using the pyuvdata package (B. Hazelton, Jacobs, Pober, \& Beardsley, 2017), which supports a variety of output data types and ensures accurate phasing methods. Source motions and coherency rotations are calculated using astropy transformations (Astropy Collaboration et al., 2013), which takes into account higher order corrections to sky motion.

Currently, pyuvsim supports simulating point sources from the GLEAM catalog (HurleyWalker et al., 2017) and several mock source catalogs. It includes utilities for making parameter files based on the structure of any data file readable by pyuvdata, allowing users to quickly set up simulations to compare with an observation. It is parallelized using the Message Passing Interface (MPI) (Dalcín, Paz, \& Storti, 2005). Performance improvements are made by identifying and correcting bottlenecks using built-in line profiling tools. Features to be added include support for spectral models for sources, diffuse source models, and ionospheric effects. These are not expected to change the API. Users can rely on pyuvsim for precise results with steady, long-term improvements in resource usage and runtime. 


\section{Acknowledgements}

This work was supported in part by NASA award 80NSSC18K0389 and NSF award AST1613973.

\section{References}

Astropy Collaboration, Robitaille, T. P., Tollerud, E. J., Greenfield, P., Droettboom, M., Bray, E., Aldcroft, T., et al. (2013). Astropy: A community Python package for astronomy. AAP, 558, A33. doi:10.1051/0004-6361/201322068

Dalcín, L., Paz, R., \& Storti, M. (2005). MPI for Python. Journal of Parallel and Distributed Computing, 65(9), 1108-1115. doi:10.1016/j.jpdc.2005.03.010

Hamaker, J. P., Bregman, J. D., \& Sault, R. J. (1996). Understanding radio polarimetry. I. Mathematical foundations. Astronomy and Astrophysics Supplement Series, 117(1), 137-147. doi:10.1051/aas:1996146

Hazelton, B., Jacobs, D., Pober, J., \& Beardsley, A. (2017). Pyuvdata: An interface for astronomical interferometeric datasets in python. The Journal of Open Source Software, 2(10), 140. doi:10.21105/joss.00140

Hurley-Walker, N., Callingham, J. R., Hancock, P. J., Franzen, T. M. O., Hindson, L., Kapińska, A. D., Morgan, J., et al. (2017). GaLactic and Extragalactic All-sky Murchison Widefield Array (GLEAM) survey - I. A low-frequency extragalactic catalogue. Monthly Notices of the Royal Astronomical Society, 464(1), 1146-1167. doi:10. 1093/mnras/stw2337

Sullivan, I. S., Morales, M. F., Hazelton, B. J., Arcus, W., Barnes, D., Bernardi, G., Briggs, F. H., et al. (2012). Fast Holographic Deconvolution: A New Technique for Precision Radio Interferometry. ApJ, 759, 17. doi:10.1088/0004-637X/759/1/17

Thyagarajan, N., Kolopanis, M., Jacobs, D., Murray, S., \& santoshmh. (2019, January). PRISim: Precision Radio Interferometry Simulator (for radio astronomy applications). Zenodo. doi:10.5281/zenodo.2548117 